\title{
BMJ Open Medication errors during simulated paediatric resuscitations: a prospective, observational human reliability analysis
}

\author{
Nicholas Appelbaum (D) ,,2,3 Jonathan Clarke (1) ,2,3,4 Calandra Feather (D) , 1,2,3 \\ Bryony Franklin, ${ }^{2,5}$ Ruchi Sinha, ${ }^{6}$ Phillip Pratt, ${ }^{3}$ Ian Maconochie, ${ }^{7}$ Ara Darzi ${ }^{1,2,3}$
}

To cite: Appelbaum N, Clarke J, Feather C, et al. Medication errors during simulated paediatric resuscitations: a prospective, observational human reliability analysis. BMJ Open 2019;9:e032686. doi:10.1136/ bmjopen-2019-032686

- Prepublication history and additional material for this paper are available online. To view these files, please visit the journal online (http://dx.doi. org/10.1136/bmjopen-2019032686).

Received 08 July 2019 Revised 23 September 2019 Accepted 17 October 2019

Check for updates

(c) Author(s) (or their employer(s)) 2019. Re-use permitted under CC BY. Published by BMJ.

For numbered affiliations see end of article.

Correspondence to Dr Nicholas Appelbaum; n.appelbaum@imperial.ac.uk

\section{ABSTRACT}

Introduction Medication errors during paediatric resuscitation are thought to be common. However, there is little evidence about the individual process steps that contribute to such medication errors in this context.

Objectives To describe the incidence, nature and severity of medication errors in simulated paediatric resuscitations, and to employ human reliability analysis to understand the contribution of discrepancies in individual process steps to the occurrence of these errors.

Methods We conducted a prospective observational study of simulated resuscitations subjected to video microanalysis, identification of medication errors, severity assessment and human reliability analysis in a large English teaching hospital. Fifteen resuscitation teams of two doctors and two nurses each conducted one of two simulated paediatric resuscitation scenarios.

Results At least one medication error was observed in every simulated case, and a large magnitude (>25\% discrepant) or clinically significant error in 11 of 15 cases. Medication errors were observed in $29 \%$ of 180 simulated medication administrations, $40 \%$ of which considered to be moderate or severe. These errors were the result of 884 observed discrepancies at a number of steps in the drug ordering, preparation and administration stages of medication use, $8 \%$ of which made a major contribution to a resultant medication error. Most errors were introduced by discrepancies during drug preparation and administration.

Conclusions Medication errors were common with a considerable proportion likely to result in patient harm. There is an urgent need to optimise existing systems and to commission research into new approaches to increase the reliability of human interactions during administration of medication in the paediatric emergency setting.

\section{INTRODUCTION}

\section{Background}

Medication errors are among the leading cause of avoidable harm in healthcare worldwide $^{1}$ and up to three times more common in children than in adults. ${ }^{2}$ The paediatric emergency environment, characterised by urgency and fraught with interruptions, is one of the clinical areas most vulnerable to error. Medication administration in emergencies is complex as it requires successful

\section{Strengths and limitations of this study}

This study adds to the literature base, highlighting paediatric emergency medication error as worrying, potentially highly harmful and requiring urgent attention.

- This study is one of the first to use Human Reliability Analysis to link task discrepancies with resultant medication errors, as well as to link these discrepancies directly to potential harm. This effort has demonstrated that a significant fraction of the burden of error in the paediatric emergency drug administration process originates during the preparation and administration phase and that most of these errors are likely to be undetected in clinical practice.

- Although we went to considerable lengths to replicate the paediatric emergency environment, the simulation environment cannot truly reflect the clinical environment during a genuine emergency.

- Furthermore, this study was conducted at a single site and participants were not blinded to the purpose of the study, so it is potentially subject to preparation bias.

- Participants were recruited from the paediatric emergency unit, intensive care unit and general paediatrics ward and had variable experience of emergency cases. However, all participants worked in clinical units that manage critically ill children.

interactions between different teams of medical and nursing staff, as well as between individual members of these teams. An additional challenge relates to obtaining relevant medication information and translating this into the required dose and concentration of the correct drug to be administered by the correct route for the clinical indication, all in a necessarily short space of time. Medication errors in general, and medication administration errors in particular, are both underdetected and under-reported, ${ }^{3}$ such that little is known of their incidence or impact during resuscitation. However, medication errors have been reported in 7 out of 10 simulated paediatric resuscitations, ${ }^{4}$ with other recent 
simulation studies suggesting $26 \%{ }^{5}$ to $70 \%{ }^{6}$ of administered medicines being given at the wrong dose. Laboratory studies analysing syringes prepared for anaesthetic use have found at least $15 \%$ to be greater than $20 \%$ discrepant from the intended drug concentration. ${ }^{7}$

The broader, systems view of medical error, heralded by the Institute of Medicine's 'To Err is Human' report, saw the widespread adoption of Reason's organisational accident model $^{8}$ in healthcare. More recently, human reliability analysis (HRA) techniques, previously commonplace only in other high risk industries, have become increasingly used in healthcare research. ${ }^{9}{ }^{10} \mathrm{HRA}$ is based on the understanding that neither humans nor systems can be error-proof, and asserts that to improve safety and reliability, a thorough analysis of system vulnerabilities at a task level is needed, taking into account human-human and human-machine interactions. ${ }^{11} 12$ Medication safety researchers have previously used an HRA technique, the systematic human error reduction and prediction approach (SHERPA), to identify system vulnerabilities in ward-based medication administration, ${ }^{13}$ anaesthesia ${ }^{14}$ and general surgery. ${ }^{15}$ This approach, however, has not been used quantitatively in medication safety research and has not been applied to paediatric resuscitation.

Our objectives were to describe the incidence, nature and severity of medication errors in simulated paediatric resuscitations, and then use HRA to understand the contributory role played by individual process step discrepancies with a focus on those discrepancies contributing to large magnitude and/or clinically significant errors.

\section{METHODS}

\section{Study design and setting}

This prospective observational study was conducted from April 2017 to November 2017 in a medical simulation facility within a large teaching hospital. The hospital has a paediatric emergency department (seeing 27000 children each year) and a comprehensive paediatric inpatient service (admitting 5000 each year). The hospital used electronic prescribing in the inpatient setting, but during resuscitations, medications were more commonly ordered on paper prescription charts. We recruited resuscitation teams of four clinicians, that were randomised to participate in one of two standardised simulated paediatric resuscitation scenarios. The study was approved by the Health Research Authority and the hospital concerned. National Health Service ethics approval was not required. Participants gave written informed consent.

\section{Patient and public involvement}

The research team held a workshop with parents to get their feedback on the proposal, develop the patient and public involvement and engagement (PPIE) plans, and identify future areas for research. We actively sought attendees through INVOLVE's 'People in Research' website, social media and Imperial College London's existing networks. Our team has also participated in a PPIE event run by the Royal College of Paediatrics and Child Health, in collaboration with MedsIQ (http://www. medsiq.org) and Medicines for Children (http://www. medicinesforchildren.org.uk), two UK-based paediatric medication safety initiatives.

\section{Participants}

Eligible participants were a convenience sample of medical and nursing staff from the departments of paediatrics and paediatric emergency medicine at the study hospital. Participants were assigned into teams comprising a senior doctor (a specialist registrar, with at least a year of prior experience as a registrar), a junior doctor, a senior nurse (with at least 5 years' nursing experience) and a junior nurse.

\section{Clinical scenarios}

The two scenarios were:

1. Prolonged status epilepticus in an 8 -month-old, $8 \mathrm{~kg}$ child.

2. Presumed meningococcal sepsis in a 10-month-old, 9 $\mathrm{kg}$ child.

The two scenarios (online supplementary appendix 1) were designed by a collaboration of paediatric nurses, emergency physicians, intensivists, general paediatricians and anaesthetists. Face validity was established by an independent expert panel of six, with representation from each of these professional groups, including two lead paediatric clinical nurse educators. It was deemed that the two scenarios were both similarly demanding and clinically sound, with treatment recommendations corresponding closely to UK Resuscitation Council and Royal College of Paediatrics and Child Health teaching cases.

A simulated paediatric resuscitation bay was created. The mannequin used was a SimBaby V.2 (Laerdal Medical, Stavanger, Norway), and the syringe pump stack consisted of Alaris PK MK4 units (Becton Dickinson, Franklin Lakes, USA). All relevant print materials (eg, British National Formulary for Children ${ }^{16}$ and local guidelines) and hospital information technology systems as well as external internet access were available. Participants were requested to prescribe, prepare and administer medications exactly as per usual practice, to use mobile applications or websites as they would in clinical practice and to telephone specialist colleagues if required. A paediatric intensivist, the hospital lead for paediatric simulation, ran the scenarios. She provided standardised clinical information as live feedback and answered questions regarding the child's response to treatment or their current condition when needed.

\section{Data sources and measurement}

A Scotia Medical Observation Training System (smots, Scotia UK, Edinburgh, UK), with two 3-axis, ceilingmounted video cameras, and three mobile, highdefinition cameras equipped with boom microphones, was used. Both nurses in each team wore head-mounted 


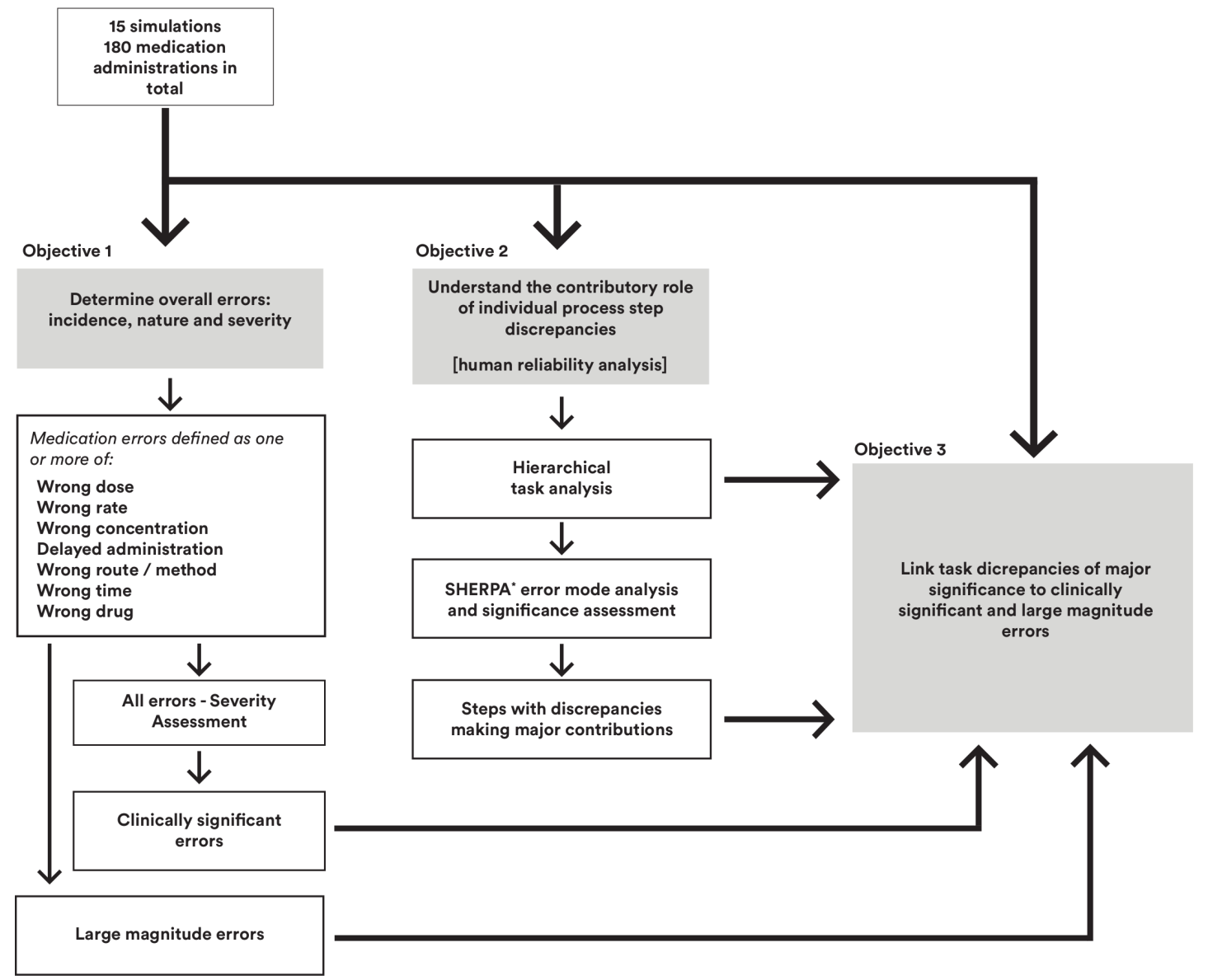

Figure 1 Overview of study objectives and analyses. *SHERPA, systematic human error reduction and prediction approach.

high-definition video cameras (GoPro Inc, California, USA). The video recordings were analysed by a research nurse with 10 years' experience in paediatric intensive care.

\section{Outcome measures}

We reserved the term 'medication error' to describe an overall error with respect to a particular drug administration as a whole, after having been administered to a patient, and the term 'discrepancy' to refer to observed deviations from expected local practice at the level of the individual task. This approach has been successfully used by other medication safety researchers. ${ }^{17}$

Variation in clinician practice and human adaption to the complex process of medication ordering, preparation and administration typically results in many minor task discrepancies that may not individually, or even in combination, result in a medication error or patient harm. ${ }^{17}$ Other discrepancies almost always result in medication errors. For example, a prescribing or pump-programming discrepancy is highly likely to result in a medication error. To identify the most important task discrepancies, we assessed all observed discrepancies to establish the extent to which they may or may not have contributed to any resultant medication errors.

Figure 1 summarises the study objectives and associated analyses.

\section{Medication errors}

Medication errors included any errors in dose, administration rate, concentration, drug, route of administration, method of administration, timing or delay in administration. Operational definitions for each of these are given in online supplementary appendix 2. Briefly, dosing errors were defined as a greater than $10 \%$ deviation from the recommended dosing range (DRDR) ${ }^{18}$ at the study site. Any deviation from the recommended rate of administration (DRDRate) was calculated in a similar manner and deviations of more than $10 \%$ were considered to be medication errors. Where there was a greater than $25 \%$ discrepancy in the DRDR or DRDRate, the errors were considered as 'large magnitude'. Deviations from the recommended concentration (DRC) of greater than $10 \%$ from the concentration specified in local guidance were also included as medication errors.

To identify delayed administrations, the time taken for the dose to be 'ready for delivery' was calculated as the time for the doctors to obtain any medication information required plus the nurse-led preparation time. The time to be ready for deliver was considered 'prolonged' when a particular team took more than double the median time for that specific drug across the entire study without clinical cause for the delay as determined by the nurse assessor. For example, if a medication administration was 
interrupted to reassess the patient clinically or to administer another medication as a priority, a prolonged time would be excluded as an error on clinical grounds.

\section{Severity assessment}

There are few validated tools that can be used to assess the potential severity of medication errors without knowledge of patient outcomes and that are thus usable in simulated studies. ${ }^{19}$ One of these tools is that of Dean and Barber, based on four to five experts independently assessing each error on a 0 to 10 scale, and their mean score used as an index of severity. Mean scores under 3 suggest errors of minor severity, those between 3 and 7 as moderate and those greater than 7 as severe. ${ }^{20}$ We used this approach, with two paediatric intensivists, one paediatric anaesthetist, one senior critical care nurse and one senior clinical pharmacist assessing each error.

\section{Discrepancies at the level of the task}

A hierarchical task analysis (HTA) was developed based on a similar framework for ward-based medication administration ${ }^{13}$ and assessed for face validity by five senior nurses in the study hospital. A generic human error taxonomy, based on the SHERPA external error modes ${ }^{21}$ with one additional error mode, 'information not sought', was used to code observed discrepancies against the HTA.
Where there were more than two discrepancies at a single step for a specific administration, the nurse assessor made a subjective assessment of which had the greater overall consequence and assigned an error mode (figure 2) to that discrepancy only. To capture 'rootcause' system vulnerabilities, steps where an action was performed correctly, but which perpetuated a previous medication error, were not classed as discrepancies. An example would be a correct volume calculation based on an incorrectly prescribed dose. In this example, the volume calculation which persisted but did not directly cause the error wouldn't be classed as a discrepancy whereas the incorrect prescription would be.

\section{Significance assessment of task discrepancies}

All task discrepancies were classified by the nurse assessor according to the contribution made by the discrepancy as follows:

No contribution: the discrepancy did not contribute to a medication error.

Minor contribution: some contribution made to a medication error.

Major contribution: the task discrepancy led directly to a medication error.

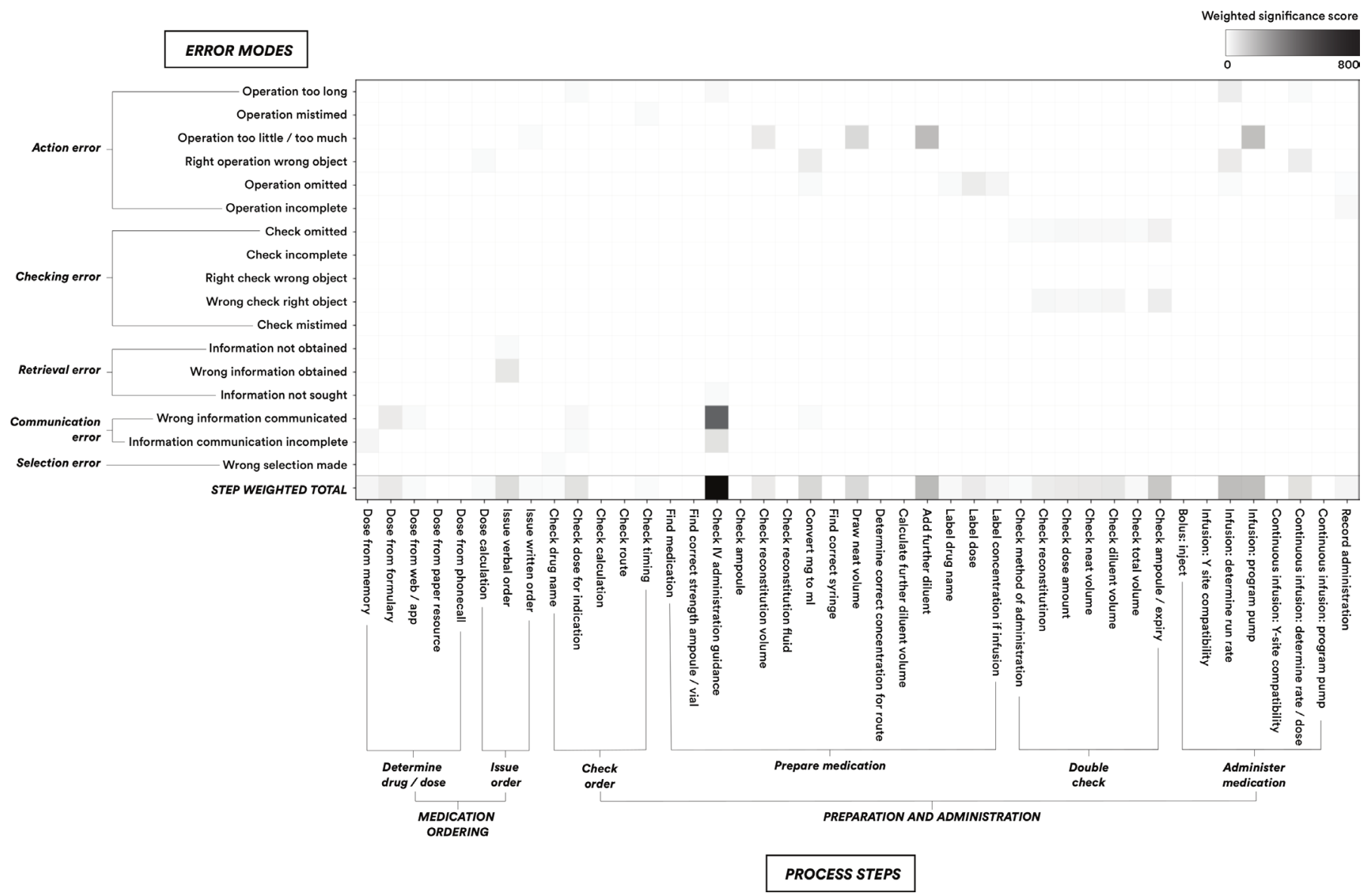

Figure 2 Significance-weighted heat-map of error by process step and SHERPA error mode. IV, intravenous; SHERPA, systematic human error reduction and prediction approach. 


\section{Data management and analysis}

Medication errors were described according to the type of error, method of administration (eg, injection, infusion, continuous infusion) and stage of the medication use process (eg, medication ordering, preparation, administration) during which the error occurred. Error rates were calculated using the total number of applicable administrations as the denominator.

Step discrepancies were presented as counts, grouped by task and contribution to medication error. Discrepancy rates were calculated as the percentage of discrepancies that made a major, minor or no contribution to an error, with the number of observed discrepancies at each process step as the denominator. Of those discrepancies making major contributions to medication errors, the proportion that led to clinically significant errors (severity score $>3$ ) and/or large magnitude errors (DRDR or DRDRate $>25 \%$ ) was also calculated.

There is no literature that quantifies the extent to which a step discrepancy having a minor or major contribution to an error is of greater significance than a step discrepancy that makes no contribution to an error. For a weighted, 'heat map' HRA analysis, it was therefore necessary to attribute different weights to discrepancies that resulted in error to those that did not. Substep discrepancies were therefore weighted, agreed by the expert panel, as follows:

- No contribution: weight $=1$

- Minor contribution: weight $=10$.

- Major contribution: weight $=40$.

The total weighted significance score for each step was thereby calculated for each error mode.

\section{Interobserver reliability}

One of the 15 simulations was reanalysed by an additional independent nurse not involved in the simulations. Spearman's rank correlation coefficient was calculated for 38 continuous variables (eg, doses, elapsed times) and Cohen's kappa for 20 categorical variables (eg, reconstitution fluid, parameters for labelling quality assessment). The data set concerning the medication errors and timing parameters was treated separately from the data set containing the parameters for the HRA (the task discrepancies).

\section{RESULTS}

Data were collected during 15 simulations according to participant availability. Eight simulations were of the prolonged seizures case, and seven of the meningococcal sepsis case. Participants comprised 30 doctors and 30 nurses (table 1), each of whom completed one simulation. For categorical variables, Cohen's kappa values were between 0.862 (medication error) and 0.954 (HRA). Continuous variables were only present in the medication error data set, for which Spearman's rank coefficient was greater than 0.904 for all variables.

\begin{tabular}{|c|c|c|c|}
\hline & Overall & Doctors & Nurses \\
\hline $\begin{array}{l}\text { Total number of } \\
\text { participants }\end{array}$ & 60 & 30 & 30 \\
\hline \multicolumn{4}{|l|}{ Age * } \\
\hline Median (range) & $30(23-51)$ & $30.5(23-44)$ & $28(23-51)$ \\
\hline \multicolumn{4}{|l|}{ Gender (\%) } \\
\hline Female & $52(87 \%)$ & $23(77 \%)$ & $29(97 \%)$ \\
\hline Male & $8(13 \%)$ & $7(23 \%)$ & $1(3 \%)$ \\
\hline \multicolumn{4}{|c|}{ Years in clinical practice (n, \%) } \\
\hline $0-5$ & $32(53 \%)$ & $11(37 \%)$ & $21(70 \%)$ \\
\hline $6-10$ & $20(33 \%)$ & $16(53 \%)$ & $4(13 \%)$ \\
\hline $11-15$ & $5(8 \%)$ & $3(10 \%)$ & $2(7 \%)$ \\
\hline $16-20$ & $1(2 \%)$ & $0(0 \%)$ & $1(3 \%)$ \\
\hline$>20$ & $2(3 \%)$ & $0(0 \%)$ & $2(7 \%)$ \\
\hline \multicolumn{4}{|c|}{ Years in paediatric practice $(n, \%)$} \\
\hline $0-5$ & $37(62 \%)$ & $16(53 \%)$ & $21(70 \%)$ \\
\hline $6-10$ & $16(27 \%)$ & $12(40 \%)$ & $4(13 \%)$ \\
\hline $11-15$ & $5(8 \%)$ & $2(7 \%)$ & $3(10 \%)$ \\
\hline $16-20$ & $1(2 \%)$ & $0(0 \%)$ & $1(3 \%)$ \\
\hline$>20$ & $1(2 \%)$ & $0(0 \%)$ & $1(3 \%)$ \\
\hline
\end{tabular}

${ }^{*}$ Age data was omitted for five participants.

\section{Medication errors}

Participants conducted 180 medication administrations. Overall, errors were observed reaching the patient for 52 drug administrations (29\%) and at least once in every simulation. Of these errors, $30(58 \%)$ were assessed as being of minor severity, $16(31 \%)$ as moderate and 6 $(12 \%)$ as severe. There were 27 large magnitude errors (52\% of all errors), in which the DRDR/DRDRate was greater than $25 \%$. Of all erroneous administrations that reached the patient, only two $(4 \%)$ were noticed by staff after administration and therefore may have been reported in clinical practice. A detailed error analysis is provided in table 2 and description of the 10 most severe errors in table 3 .

\section{Hierarchical task analysis}

The full HTA is shown as figure 3 and shows all steps assessed in the paediatric emergency drug administration process.

\section{Errors and discrepancies by stage of medication use and process substep}

Overall, 884 step discrepancies were observed, excluding dependent downstream discrepancies after an initial discrepancy. Of these 884 step discrepancies, 174 (20\%) were linked to a medication error, with $70(8 \%)$ assessed as making a major contribution to an error, $104(12 \%)$ making a minor contribution and $710(80 \%)$ making no contribution. 
Table 2 Incidence, nature and severity of errors, presented by phase and type of error and method of administration

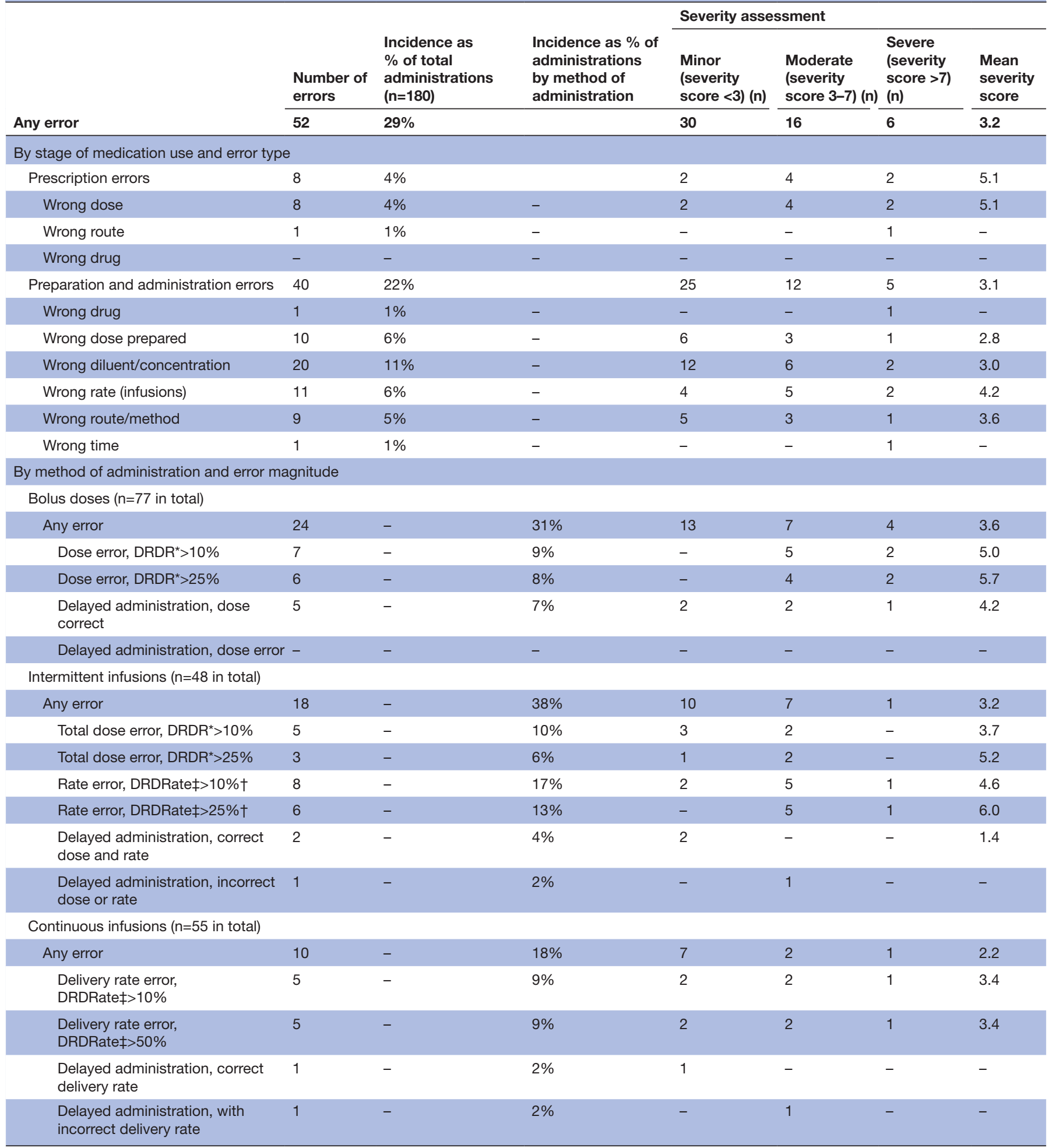

More than one error type can occur in one medication administration and a single medication error may meet more than one criterion, so individual error types do not sum to the total by stage or method. Other error types (for example diluent errors) are included in the 'any error' counts but are not presented as sub-counts.

${ }^{*}$ DRDR (deviation from recommended dosing range)=absolute value of the percentage difference from the recommended dose or dose range. †Rate errors are only shown for intermittent infusions where the delivery rate error is due to a pump-programming error.

łDRDRate (deviation from recommended dosing rate)=absolute value of the percentage difference from the recommended rate of administration.

Figure 2 shows the significance-weighted HRA data represented as a heat-map demonstrating the relative contributions of discrepancies at each step and by each error mode to medication errors. Table 4 summarises the discrepancy counts per step as well as the percentage of both large magnitude and clinically 


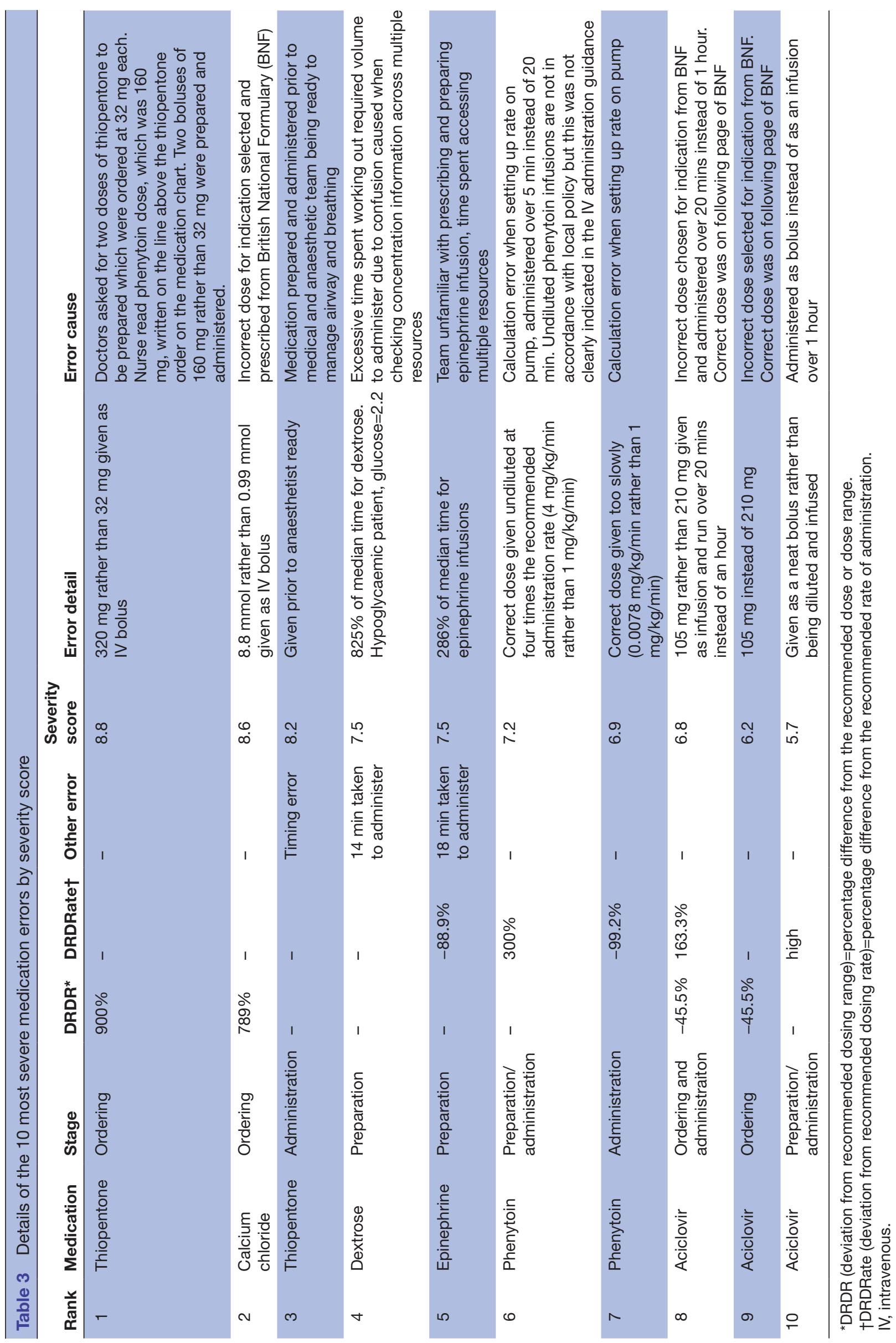




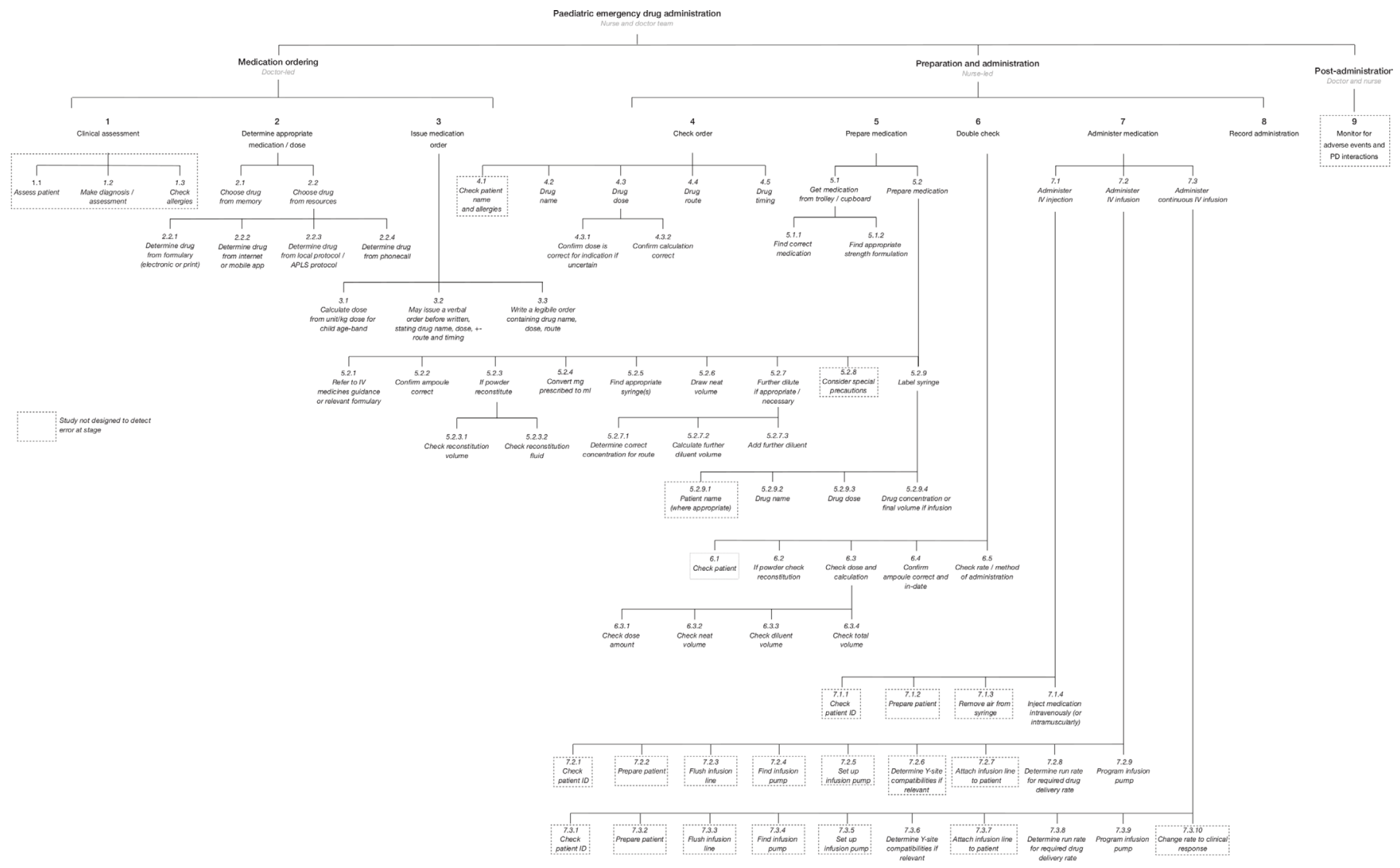

Figure 3 The hierarchical task analysis.

significant errors with a major contribution made at each HTA step.

\section{Errors and discrepancies during medication ordering}

We observed 170 discrepancies during the ordering phase. Five of the 22 clinically significant medication errors were due to discrepancies during medication ordering, with three of these due to incorrect dose information retrieval from the British National Formulary for Children. The majority of the remaining discrepancies (136) were due to incomplete verbal medication orders based on which drug preparation commenced, two of which resulted in medication errors. Of the 180 written medication orders examined, there were six discrepancies observed, all of which were corrected by the clinicians and therefore made no contribution to any dosing errors, but did result in one delayed administration.

\section{Errors and discrepancies during medication preparation}

During medication preparation, 310 discrepancies were observed, representing $35 \%$ of all observed discrepancies. These contributed to one medication error involving the wrong drug, 10 dose errors and 20 diluent or dilution errors. The retrieval of preparation and administration information from the online intravenous medications guide was the step mostly likely to contribute to medication errors during medication preparation, with 42 discrepancies (19 major contribution to a medication error, one minor), resulting in nine clinically significant medication errors (severity score $>3$ ). The retrieval of incorrect information and taking an excessively long time to identify the correct information within the guidance were the most common discrepancy error modes.

There were seven discrepancies when converting milligrams to millilitres of undiluted drug, and 18 discrepancies (six making a major contribution) when, after having made the correct calculations, nurses withdrew either the incorrect amount of undiluted drug or the incorrect amount of diluent into the syringe.

\section{Double-checking}

Overall, 259 discrepancies were observed during the double-checking phase, 72 (28\%) of which made a minor contribution to a medication error. Checking the route and method (eg, infusion or bolus) of administration was the most frequently omitted.

We observed 29 medication errors that were made during medication ordering and preparation but had not been yet administered to the patient at the point of double-checking. These errors were thus potentially 'interceptable' but all ultimately reached the patient. Of these errors, in 14 cases, the double-checking interaction between the nurses included the incorrect step but failed to identify it as incorrect.

Errors and discrepancies during the administration phase Of all observed discrepancies, only 28 (3\%) occurred during administration. These resulted in 11 wrong rate 
Table 4 Number, frequency and relationship of discrepancies to resultant errors with subanalysis of major discrepancies that resulted in large magnitude and clinically significant errors

\section{Relationship to resultant errors}

Discrepancies which made a major contribution to a medication error

\begin{tabular}{|c|c|c|c|c|c|c|}
\hline Stage of deviation & $\begin{array}{l}\text { Total } \\
\text { discrepancies } \\
\text { (n) }\end{array}$ & $\begin{array}{l}\text { No contribution } \\
\text { n (\%*) }\end{array}$ & $\begin{array}{l}\text { Minor } \\
\text { contribution n } \\
\left(\%{ }^{*}\right)\end{array}$ & $\begin{array}{l}\text { Major } \\
\text { contribution, } \\
\text { total (n, \%*) }\end{array}$ & $\begin{array}{l}\text { Discrepancies } \\
\text { that resulted } \\
\text { in clinically } \\
\text { significant errors } \\
\left(n, \%^{*}\right) \dagger\end{array}$ & $\begin{array}{l}\text { Discrepancies } \\
\text { that resulted in } \\
\text { large magnitude } \\
\text { errors } n\left(\%^{*}\right) \ddagger\end{array}$ \\
\hline Overall & 884 & $710(80)$ & $104(12)$ & $70(8)$ & $33(47)$ & $31(44)$ \\
\hline Ordering phase & 170 & $159(94)$ & $2(1)$ & $9(5)$ & $5(23)$ & $6(22)$ \\
\hline Determine dose & 29 & $21(72)$ & $1(3)$ & $7(24)$ & $5(23)$ & $6(22)$ \\
\hline Dose from formulary & 10 & $6(60)$ & $1(10)$ & $3(30)$ & $3(14)$ & $3(11)$ \\
\hline Dose from other resource & 11 & $10(91)$ & - & $1(9)$ & $1(5)$ & $1(4)$ \\
\hline Dose calculation & 4 & $3(75)$ & - & $1(25)$ & $1(5)$ & $1(4)$ \\
\hline Issue order & 141 & $138(98)$ & $1(1)$ & $2(1)$ & - & - \\
\hline Check drug name & 1 & - & - & $1(100)$ & $1(5)$ & $1(4)$ \\
\hline Check dose for indication & 15 & $5(33)$ & $8(53)$ & $2(13)$ & $1(5)$ & - \\
\hline Check calculation & 1 & - & $1(100)$ & - & - & - \\
\hline Check route and timing & 2 & $1(50)$ & - & $1(50)$ & $1(5)$ & - \\
\hline Preparation, actual & 310 & $263(85)$ & $8(3)$ & $39(13)$ & $14(64)$ & $12(44)$ \\
\hline $\begin{array}{l}\text { Find correct medication } \\
\text { and strength of vial }\end{array}$ & 2 & - & $2(100)$ & - & - & - \\
\hline $\begin{array}{l}\text { Check intravenous } \\
\text { administration guidance }\end{array}$ & 42 & $22(52)$ & $1(2)$ & $19(45)$ & $9(41)$ & $6(22)$ \\
\hline $\begin{array}{l}\text { Determine correct } \\
\text { concentration for route }\end{array}$ & 2 & $2(100)$ & - & - & - & - \\
\hline $\begin{array}{l}\text { Calculate further diluent } \\
\text { volume and dilute }\end{array}$ & 12 & $4(33)$ & $1(8)$ & $7(58)$ & $1(5)$ & $1(4)$ \\
\hline $\begin{array}{l}\text { Label syringe with } \\
\text { drug name, dose } \\
\text { and concentration if } \\
\text { appropriate }\end{array}$ & 231 & $227(98)$ & $4(2)$ & - & - & - \\
\hline Double checking & 259 & $187(72)$ & $72(27)$ & - & - & - \\
\hline Check reconstitution & 9 & $5(57)$ & $4(44)$ & - & - & - \\
\hline $\begin{array}{l}\text { Check dose and neat } \\
\text { volume }\end{array}$ & 69 & $48(70)$ & $21(30)$ & - & - & - \\
\hline $\begin{array}{l}\text { Check diluent volume and } \\
\text { total volume separately }\end{array}$ & 86 & $63(73)$ & $23(27)$ & - & - & - \\
\hline Check ampoule+expiry & 29 & $26(90)$ & $3(10)$ & - & - & - \\
\hline Check rate/method & 66 & $45(68)$ & 21 (32) & - & - & - \\
\hline
\end{tabular}

Continued 


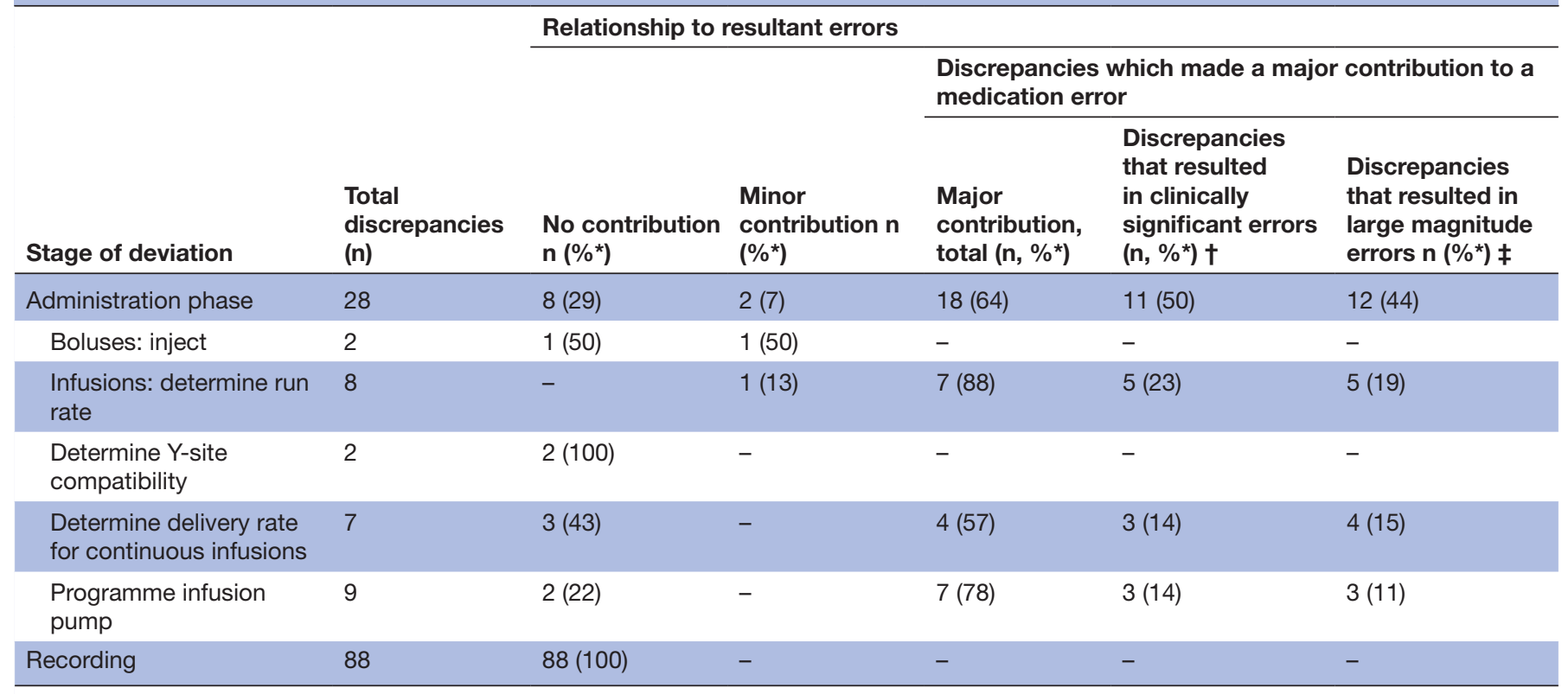

*The denominator for percentages is the total number of discrepancies in each row.

†Number and percentage of clinically significant errors (severity score $>3$ ) with major contributory discrepancies made at each specific step, of a total of 22 clinically significant errors.

¥Number and percentage of large magnitude errors (deviation from recommended dosing range (DRDR) or deviation from recommended dosing rate (DRDRate) $>25 \%$ ) with major contributory discrepancies made at each specific step, of a total of 27 large magnitude errors.

errors (five moderate, two severe), nine wrong method errors (three moderate, one severe) and one severe wrong time error. Discrepancies during the administration phase constituted a third of all discrepancies that made a major contribution to a clinically significant error.

Infusions in particular were prone to administration errors. Of the 17 discrepancies observed during infusion rate calculations or when programming the infusion pump for intermittent infusions, 14 were of major consequence, and accounted for $23 \%$ of all clinically severe errors. Seven discrepancies (four making a major contribution to a medication error) occurred when determining the delivery rate for continuous infusions.

\section{DISCUSSION}

This prospective observational study is the first in paediatric emergency medicine to include a quantitative HRA, allowing identification of the task discrepancies making the greatest contribution to medication error. We identified at least one medication error in all 15 simulations, and a large magnitude or clinically significant error in 12 of these.

\section{Comparison with previous literature}

Historical heterogeneity of the definitions of medication error and the variability in reporting metrics make comparison with previous literature difficult. ${ }^{22}{ }^{23}$ Additionally, there are few simulated studies and no relevant clinical studies in paediatric resuscitation, making comparator data scarce.

Prescribing error rates in the (non-simulated) emergency setting have been reported to be between $10.1 \%$ and $16 \% 2425$ of all orders; our study reports a lower rate of $5 \%$, although this difference may be at least partly due to different error definitions. Our study reinforces similar findings in another recent analysis ${ }^{26}$ suggesting that preparation and administration errors may be more common, but is the first to highlight the extent to which these errors go undetected. Other simulated studies have reported error rates for the administration of intravenous bolus medication of between $15.5 \%$ and $26.5 \%{ }^{4527}$; in our study, it was $31 \%$. The referenced studies, however, reported only on dose errors, and not any other medication error types. Only seven out of the 24 medication errors we observed for bolus doses were dose errors. Medications given by intermittent infusion were the most error-prone in our study. There are no studies that investigate emergency administration of intermittent infusions in sufficient detail to provide a basis against which to compare this finding.

Medications given by continuous infusion are potentially the most complicated in paediatric emergencies. In addition to the preparation steps for intermittent infusions, staff generally have to convert infusion rates in milligrams or micrograms per kilogram per minute to infusion rates of millilitres per hour. A recent trial of a digital application reported errors in $70 \%$ of continuous vasopressor infusions ${ }^{6}$ in the control arm. However, despite the increased cognitive demand, we observed the lowest incidence of medication error for continuous infusions, at $18 \%$. Administration of continuous infusions in our hospital seemed to be relatively well supported by an online/paper tool. ${ }^{28}$ 


\section{Limitations}

Although considerable efforts were made to replicate the paediatric emergency department environment in a state-of-the-art facility, the simulation environment may not reflect the clinical environment during a genuine emergency. Participants were not blinded to the purpose of the study, and therefore this investigation is potentially subject to preparation bias.

This investigation was conducted at a single site and all three of the nurse video assessors were from the same academic unit in the study hospital. It is thus possible that, despite best efforts at standardisation, the results may be skewed to reflect the expectations of medication practice at the study site.

Although this is one of the largest simulations studies in paediatric emergency medicine investigating medication errors, the sample size made it impossible to make probabilistic assessments of the relationship between step discrepancies and medication errors. Furthermore, the discrepancy assessment was made by a single nurse assessor. Save for the extract on which inter-rater reliability was tested, we did not have the resources to evaluate 884 discrepancies by more than one clinician, reaching consensus on each.

Finally, we did not investigate the potential role of clinical pharmacists in the resuscitation setting. There is convincing evidence that the presence of clinical pharmacists reduces medication errors in this setting, ${ }^{29-31}$ and the emergency department at the study site does, at times, benefit from the assistance and expertise of clinical pharmacists. However, their presence is not routine during resuscitations.

\section{Implications for research and practice}

This study highlights the need for research to optimise clinicians' use of electronic resources containing medication preparation and administration information. We were not able to pinpoint the precise steps at which the current electronic intravenous medications guidance system in the study hospital proved vulnerable to misinterpretation. Research to further understand the steps that need attention may serve as a useful basis from which to refine, and if needed, redesign such systems.

This study reaffirms that performing complex arithmetic in high-stress clinical environments is a considerable contributor to medication error. ${ }^{32}$ With the purpose of addressing medication safety in paediatric resuscitation, the literature has been dominated by studies looking at 'resuscitation aids', most commonly length-based tapes. ${ }^{1833} 34$ These aids couple weight estimation with a suggested dose for a limited number of medications, but usually do not provide comprehensive preparation and administration support. It is not likely that length-based tapes would have decreased the rate of medication error in this study. Further clinical research is required to determine the effectiveness of new digital tools that do support preparation and administration, such as those that have shown promising results in simulated studies. ${ }^{635}$
Human factors methods have been used in other high-risk industries to define system vulnerabilities for building safer systems. By using quantitative HRA, this study provides evidence for the prioritisation of research efforts directed towards new interventions to address the most important system weaknesses.

In terms of implications for practice, one of the most unexpected findings in this investigation was the uncovering of 'purely mechanical' task discrepancies resulting in medication errors. During drug preparation, clinicians were observed drawing up incorrect volumes of medications or diluents even though all calculations were correct. This suggests that efforts seeking to address medication safety in cognitively demanding environments using clinical education strategies or contemporary technologies must do so without disregarding the seemingly 'simplest' aspects of drug preparation. The reliability of information exchanges between healthcare professionals similarly needs improvement. Verbal medication orders in particular are inconsistent and error-ridden. Particular attention should be paid to medication orders given verbally in the emergency setting, using approaches such as the recipient verbally confirming the medication and dose being prepared. More importantly however, there is an urgent need for research to explore how to bring greater effectiveness to checking and double-checking more broadly. These are steps intended to defend patients from error, but which are too often ineffective.

\section{CONCLUSIONS}

Overall, we identified errors in $29 \%$ of all simulated medication administrations, only two of which were detected by participants, with $40 \%$ of these likely to result in moderate or severe harm. HRA revealed a number of error-prone steps, many of which occurred during preparation and administration of correctly ordered medications. The task most likely to result in erroneous medication administration was ineffective retrieval of correct medication preparation and administration instructions from intravenous medication guidance.

This study has highlighted an urgent need to optimise existing systems and to commission new approaches to increase the reliability of human interactions with the emergency medication administration process.

\section{Author affiliations}

${ }^{1}$ Department of Surgery and Cancer, Division of Surgery, Imperial College London, London, UK

${ }^{2}$ NIHR-Imperial Patient Safety Translational Research Centre, Imperial College London, London, UK

${ }^{3}$ Helix Centre for Design in Healthcare, Imperial College London, London, UK

${ }^{4}$ Centre for Mathematics of Precision Healthcare, Imperial College London, London, UK

${ }^{5}$ School of Pharmacy, University College London, London, UK

${ }^{6}$ Department of Paediatric Intensive Care, Division of Women and Children's Services, Imperial College Healthcare NHS Trust, London, UK

${ }^{7}$ Department of Emergency Medicine, Division of Medicine, Imperial College London, London, UK 


\section{Twitter Nicholas Appelbaum @nicapples}

Acknowledgements We would like to thank all clinical staff who participated in the simulations. It is our hope that the enthusiasm and extraordinary openmindedness by our dedicated and deeply competent colleagues demonstrated in making this study possible will lead to safer systems and innovations in paediatric medication safety. This study was funded by the NIHR-Imperial Patient Safety Translational Research Centre, with infrastructure support from the NIHR Imperial Biomedical Research Centre. Support was also received from the Resuscitation Council (UK).

Contributors NA, CF, JC and RS designed the study. RS facilitated the simulations, with $N A$ and $C F$ observing and coordinating. AD provided funding. CF analysed the video footage. NA and $\mathrm{JC}$ were responsible for the data analysis which was reviewed by PP. BF and IM advised on error definitions and analysis. NA drafted the study report, which was reviewed by all authors. All authors read and approved the final draft.

Funding NA received funding from the Resuscitation Council (UK) which partially funded elements of the simulations and data extraction, with the majority of the funding received from the National Institute for Health Research (NIHR) Imperial Patient Safety Translational Research Centre. Infrastructure support was provided by the NIHR Imperial Biomedical Research Centre (BRC). BF is also supported by the NIHR Health Protection Research Unit in Healthcare Associated Infections and Antimicrobial Resistance at Imperial College London, in partnership with Public Health England (PHE). The views expressed are those of the authors and not necessarily those of the NHS, PHE, the NIHR or the Department of Health and Socia Care.

Competing interests The Helix Centre at Imperial College London is leading an effort in collaboration with the British National Formulary developing digital tools in an attempt to improve paediatric medication safety. NA has written two patents describing syringe labelling techniques in medication safety. BF supervises a PhD student part funded by a supplier of hospital electronic health record systems and has received funding from Pfizer for organising and chairing two symposia on medication safety. We confirm that we have given due consideration to the protection of intellectual property associated with this work and that there are no impediments to publication, including the timing of publication, with respect to intellectual property. In so doing we confirm that we have followed the regulations of our institution concerning intellectual property.

Patient consent for publication Not required.

Ethics approval The Health Research Authority approved this study.

Provenance and peer review Not commissioned; externally peer reviewed.

Data availability statement Data are available upon reasonable request.

Open access This is an open access article distributed in accordance with the Creative Commons Attribution 4.0 Unported (CC BY 4.0) license, which permits others to copy, redistribute, remix, transform and build upon this work for any purpose, provided the original work is properly cited, a link to the licence is given, and indication of whether changes were made. See: https://creativecommons.org/ licenses/by/4.0/.

ORCID iDs

Nicholas Appelbaum https://orcid.org/0000-0002-9458-6828

Jonathan Clarke https://orcid.org/0000-0003-1495-7746

Calandra Feather http://orcid.org/0000-0003-1322-6589

\section{REFERENCES}

1 World Health Organization. Medication without harm. B World Health Organ 2017;95:317.

2 Wong ICK, Ghaleb MA, Franklin BD, et al. Incidence and nature of dosing errors in paediatric medications: a systematic review. Drug Saf 2004;27:661-70.

3 Allan EL, Barker KN. Fundamentals of medication error research. Am J Hosp Pharm 1990;47:555-71.

4 Kozer E, Seto W, Verjee Z, et al. Prospective observational study on the incidence of medication errors during simulated resuscitation in a paediatric emergency department. BMJ 2004;329.

5 Moreira ME, Hernandez C, Stevens AD, et al. Color-Coded prefilled medication syringes decrease time to delivery and dosing error in simulated emergency department pediatric resuscitations. Ann Emerg Med 2015;66:97-106.

6 Siebert JN, Ehrler F, Combescure C, et al. A mobile device APP to reduce time to drug delivery and medication errors during simulated pediatric cardiopulmonary resuscitation: a randomized controlled trial. J Med Internet Res 2017;19:e31.

7 Stucki C, Sautter A-M, Wolff A, et al. Accuracy of preparation of i.v. medication syringes for anesthesiology. Am J Health Syst Pharm 2013;70:137-42.

8 Reason J. Human error. Cambridge: University of Cambridge, 1990.

9 Embrey D. Application of SHERPA to predict and prevent use error in medical devices. paper presented at: International Symposium on human factors and ergonomics in healthcare: advancing the cause, 2014: 246-53.

10 Lyons MAS, Woloshynowych M, Vincent C. Human reliability analysis in healthcare: a review of techniques. Int J Risk Saf Med 2004;16:223-37

11 Kirwan BAL. A guide to task analysis. London: Taylor and Francis, 1992.

12 National Offshore Petroleum Safety and Environmental Management Authority. Human reliability analysis, 2018. Available: https://www. nopsema.gov.au/resources/human-factors/human-reliability-analysis/

13 Lane R, Stanton NA, Harrison D. Applying hierarchical task analysis to medication administration errors. Appl Ergon 2006;37:669-79.

14 Phipps D, Meakin GH, Beatty PCW, et al. Human factors in anaesthetic practice: insights from a task analysis. Br J Anaesth 2008;100:333-43.

15 Joice P, Hanna GB, Cuschieri A. Errors enacted during endoscopic surgery-a human reliability analysis. Appl Ergon 1998;29:409-14.

16 BMJ Group and Royal Pharmaceutical Socety. British National formulary for children. London: Pharmaceutical Press, 2018-2019.

17 Lyons I, Furniss D, Blandford A, et al. Errors and discrepancies in the administration of intravenous infusions: a mixed methods multihospital observational study. BMJ Qual Saf 2018;27:892-901.

18 Shah AN, Frush K, Luo X, et al. Effect of an intervention standardization system on pediatric dosing and equipment size determination: a crossover trial involving simulated resuscitation events. Arch Pediatr Adolesc Med 2003;157:229-36.

19 Garfield S, Reynolds M, Dermont L, et al. Measuring the severity of prescribing errors: a systematic review. Drug Saf 2013;36:1151-7.

20 Dean BS, Barber ND. A validated, reliable method of scoring the severity of medication errors. Am J Health Syst Pharm 1999;56:57-62.

21 Embrey D. A systematic human error reduction and prediction approach. Paper presented at: International topical meeting advances in human factors in nuclear power systems. La Grange Park, KnoxvilleTennessee, IL: American Nuclear Society, 1986.

22 McLeod MC, Barber N, Franklin BD. Methodological variations and their effects on reported medication administration error rates. $B M J$ Qual Saf 2013;22:278-89.

23 Lisby M, Nielsen LP, Brock B, et al. How are medication errors defined? A systematic literature review of definitions and characteristics. Int J Qual Health Care 2010;22:507-18.

24 Kozer E, Scolnik D, Macpherson A, et al. Variables associated with medication errors in pediatric emergency medicine. Pediatrics 2002;110:737-42.

25 Jain S, Basu S, Parmar VR. Medication errors in neonates admitted in intensive care unit and emergency department. Indian J Med Sci 2009;63:145-51.

26 Murugan S, Parris P, Wells M. Drug preparation and administration errors during simulated paediatric resuscitations. Arch Dis Child 2019;104:444-450.

27 Porter E, Barcega B, Kim TY. Analysis of medication errors in simulated pediatric resuscitation by residents. West J Emerg Med 2014;15:486-90.

28 Children's Acute Transfer Service. Electronic drug chart for referring hospitals. Available: http://site.cats.nhs.uk/wp-content/uploads/ 2014/11/dgh drugcalculator v1.2.pdf

29 Patanwala AE, Sanders AB, Thomas MC, et al. A prospective, multicenter study of pharmacist activities resulting in medication error interception in the emergency department. Ann Emerg Med 2012;59:369-73.

30 Rothschild JM, Churchill W, Erickson A, et al. Medication errors recovered by emergency department pharmacists. Ann Emerg Med 2010;55:513-21.

31 Aldridge VE, Park HK, Bounthavong M, et al. Implementing a comprehensive, 24-hour emergency department pharmacy program. Am J Health Syst Pharm 2009;66:1943-7.

32 Potts MJ, Phelan KW. Deficiencies in calculation and applied mathematics skills in pediatrics among primary care interns. Arch Pediatr Adolesc Med 1996;150:748-52.

33 Fineberg SL, Arendts G. Comparison of two methods of pediatric resuscitation and critical care management. Ann Emerg Med 2008:52:e13:35-40. 
34 Campagne DD, Young M, Wheeler J, et al. Pediatric tape: accuracy and medication delivery in the National Park service. West J Emerg Med 2015;16:665-70.
35 Damhoff HN, Kuhn RJ, Baker-Justice SN. Medication preparation in pediatric emergencies: comparison of a web-based, standarddose, bar code-enabled system and a traditional approach. J Pediatr Pharmacol Ther 2014;19:174-81. 